\title{
Metabolically Abnormal Obesity Increases the Risk of Advanced Prostate Cancer in Chinese Patients Undergoing Radical Prostatectomy
}

This article was published in the following Dove Press journal: Cancer Management and Research

\author{
Wen Liu' \\ Wenxian $\mathrm{Li}^{\prime}$ \\ Zhankun Wang ${ }^{2}$ \\ Yao Zhu ${ }^{3}$ \\ Dingwei $\mathrm{Ye}^{3}$ \\ Guiming Zhang $\mathbb{D D}^{\prime}$ \\ 'Department of Urology, The Affiliated \\ Hospital of Qingdao University, Qingdao, \\ People's Republic of China; ${ }^{2}$ Department \\ of Urology, Qingdao Eighth People's \\ Hospital, Qingdao, People's Republic of \\ China; ${ }^{3}$ Department of Urology, Fudan \\ University Shanghai Cancer Center, \\ Shanghai, People's Republic of China
}

Background: To investigate the pathological risk of prostate cancer ( $\mathrm{PCa}$ ) according to the obesity and metabolic status of Chinese patients undergoing radical prostatectomy.

Materials and Methods: We performed a retrospective cross-sectional study of 1016 patients with PCa who underwent radical prostatectomy and whose metabolic status and body mass index were examined. Multivariate logistic regression analysis was performed to examine the relationship between different metabolic obesity phenotypes and the pathological outcomes of PCa.

Results: Among 1016 men, 551 (54.2\%), 106 (10.4\%), 238 (23.4\%), and 121 (11.9\%) were assigned to the metabolically healthy and normal weight (MHNW) group, metabolically abnormal but normal weight (MANW) group, metabolically healthy but overweight or obese (MHO) group, and metabolically abnormal and overweight or obese (MAO) group, respectively. Compared with the MHNW group, the MAO group had a significantly greater risk of a higher prostatectomy Gleason score [odds ratio (OR), 1.907; 95\% confidence interval ( $95 \%$ CI), 1.144-3.182], pathological stage (OR, 1.606; 95\% CI, 1.035-2.493), and seminal vesicle invasion (OR, 1.673; 95\% CI, 1.041-2.687). In contrast, the ORs were not increased in the MHO or MANW group. In the context of normal weight, metabolic disorders were associated with lymph node involvement. The metabolic status and body mass index were not associated with extracapsular extension or surgical margins in any of the four groups.

Conclusion: The MAO phenotype is associated with aggressive $\mathrm{PCa}$, including a higher prostatectomy Gleason score, pathological stage, and seminal vesicle invasion and might also be associated with disease progression. Obesity and metabolic disorders act synergistically to increase the pathological risk of PCa.

Keywords: prostate cancer, obesity, metabolic syndrome, pathology, Gleason score

\section{Introduction}

Prostate cancer (PCa) was the second most common malignancy and the fifth leading cause of cancer-related death among males worldwide in 2018 . $^{1}$ Since the westernization of the Chinese lifestyle and the increased use of prostate-specific antigen (PSA) screening, PCa has become the sixth most common cancer and the ninth most common cause of cancer-related death among Chinese men, with an especially high incidence in urban areas. ${ }^{2,3}$ The etiology and pathogenesis of PCa are complicated and still unclear. Many exogenous and environmental factors have been connected with the occurrence and progression of $\mathrm{PCa}$, including the genetic background, dietary factors, and metabolic syndrome (MetS) along with its components such as obesity, hypertension, diabetes, and dyslipidemia. ${ }^{3,4}$
Correspondence: Guiming Zhang Department of Urology, The Affiliated Hospital of Qingdao University, No. 16, Jiangsu Road, Qingdao 266003, People's Republic of China

Tel +86-532 82913056

Fax +86-532 82911999

Email zhangguiming9@126.com 
A large-scale research project in Scandinavia showed a positive association between MetS and an elevated risk of PCa. ${ }^{5}$ Obesity, diabetes, hypertension, and dyslipidemia have all been proven to be associated with an increased risk of PCa. ${ }^{6-10}$ In addition, several studies have investigated the relationship between MetS and worsening of the pathological features of $\mathrm{PCa}^{3,11,12}$ For example, our previous studies showed that MetS and dyslipidemia could predict advanced PCa in Chinese patients. ${ }^{3,13}$ Obesity is closely associated with many metabolic abnormalities, and most obese individuals have one or more metabolic disorders. However, approximately $10 \%$ to $25 \%$ of the obese individuals do not have obesity-related metabolic abnormalities and are often referred to as having metabolically healthy obesity (MHO). Conversely, some people without obesity also have some form of metabolic abnormalities. The subgroups of people with $\mathrm{MHO}$ and those with obesity-related metabolic abnormalities have become of interest. Numerous studies have shown that individuals with MHO have a lower risk of cardiovascular disease (CVD) and mortality than individuals with metabolically abnormal obesity and a higher risk than normal-weight individuals. ${ }^{14}$ Several studies have explored the relationship of different metabolic obesity phenotypes with obesity-associated cancers, such as colorectal and breast cancers. ${ }^{15,16}$ The metabolically abnormal but normal weight (MANW) and metabolically abnormal obesity (MAO) phenotypes are associated with an increased risk of breast cancer in postmenopausal women and an increased risk of advanced colorectal adenoma. ${ }^{15,17,18}$ However, no study to date has focused on the effects of different metabolic obesity phenotypes on $\mathrm{PCa}$ progression. In this study, we examined whether a relationship exists between different metabolic obesity phenotypes and the pathological characteristics of PCa among Chinese men.

\section{Patients and Methods}

\section{Patient Selection}

In total, 1597 consecutive patients with clinically localized $\mathrm{PCa}$ from 2 clinical centers were enrolled in this retrospective study: the Department of Urology at the Affiliated Hospital of Qingdao University from January 2011 to June 2014, and the Department of Urology at Fudan University Shanghai Cancer Center from January 2005 to June 2014. Radical prostatectomy and standard pelvic lymphadenectomy were performed in all patients. We excluded patients who received neoadjuvant therapy and 202 patients with missing lipid profile data. Finally, 1016 patients were included in this study. The study protocol was approved by the institutional research review boards of the two institutions. All participants provided written informed consent, and all samples were anonymous.

The following clinicopathological data were collected from the patients' medical reports: age, smoking status, height, weight, preoperative PSA level, biopsy Gleason score (GS), clinical stage (TNM classification), prostatectomy GS, pathological stage, lymph node involvement (LNI), seminal vesicle invasion, extracapsular extension, and surgical margin. Laboratory evaluation (measurement of serum glucose level and lipid profile) was performed using standard methods after overnight fasting.

\section{Definitions of Metabolic Disorders and Obesity}

The patients' metabolic status was defined according to the criteria recommended by the Chinese Diabetes Society. ${ }^{19}$ Participants with two or more of the following four parameters were defined as having a metabolically abnormal state, and those with one or no metabolic components were defined as metabolically healthy: 1) high fasting plasma glucose level of $\geq 6.1 \mathrm{mmol} / \mathrm{L}$ and/or drug treatment for an elevated glucose level, 2) high blood pressure (systolic blood pressure of $\geq 130 \mathrm{mmHg}$ and/or diastolic blood pressure of $\geq 85 \mathrm{mmHg}$ ) and/or antihypertensive drug treatment in a patient with a history of hypertension, 3) high triglyceride level of $\geq 1.7 \mathrm{mmol} / \mathrm{L}$ and/or drug treatment for a high triglyceride level, and 4) low high-density lipoprotein cholesterol level of $<40 \mathrm{mg} / \mathrm{dL}$ and/or drug treatment for a low high-density lipoprotein cholesterol level. A body mass index (BMI) cutoff point of $25 \mathrm{~kg} /$ $\mathrm{m}^{2}$ was used to define overweight/obesity $\left(\geq 25 \mathrm{~kg} / \mathrm{m}^{2}\right)$ or normal weight $\left(<25 \mathrm{~kg} / \mathrm{m}^{2}\right)$ according to the World Health Organization. ${ }^{20}$

To assess the independent contributions of adiposity and metabolic abnormalities to the risk of $\mathrm{PCa}$, we crossclassified participants according to categories of the BMI and metabolic status, yielding four phenotypes: 1) metabolically healthy and normal weight (MHNW) group, 2) MANW group, 3) MHO group, and 4) MAO group.

\section{Statistical Analyses}

Differences among the categorical variables were evaluated using the $\chi^{2}$ test. Continuous variables were analyzed using one-way analysis of variance. Multiple logistic regression analyses were performed to identify the impact 
of different categories of obesity and metabolic status on the pathological characteristics of $\mathrm{PCa}$ by odds ratios (ORs) and 95\% confidence intervals (95\% CIs). All reported $P$ values were two-sided, and $P$ values of $<0.05$ were considered statistically significant. Statistical analyses were performed using SPSS v.16.0 (SPSS Inc., Chicago, IL, USA).

\section{Results}

In total, 1016 patients were included in this study. They were diagnosed with $\mathrm{PCa}$ at a median age of 68 years (range, 41-79 years). The baseline characteristics of the four groups are shown in Table 1. Of the 1016 men, 551 (54.2\%), 106 (10.4\%), 238 (23.4\%), and 121 (11.9\%) were assigned to the MHNW, MANW, MHO, and MAO groups, respectively. No significant differences in age, smoking status, biopsy GS, or clinical stage were found among the four groups. However, the PSA level was significantly different among the groups, ranging from 1.13 to 303.00 $\mathrm{ng} / \mathrm{mL}$ (median, $14.82 \mathrm{ng} / \mathrm{mL}$ ). The MAO group had a higher PSA level.

The pathological features of $\mathrm{PCa}$, including the prostatectomy GS, pathological stage, and seminal vesicle invasion, were associated with the classification of obesity and metabolic status $(P=0.063,0.005$, and 0.005 , respectively). Figure 1 shows the percentages of the prostatectomy GS, pathological stage, and seminal vesicle invasion in different metabolic obesity phenotypes. The data demonstrated a trend of decreasing proportion in the pathological stage and seminal vesicle invasion. The proportion of each pathological feature in the MAO group was highest among the four groups. The prostatectomy GS was significantly different between the normal and abnormal weight groups, but metabolic abnormalities had no additional effect in the normal weight group: MHNW (26.32\%), MANW (27.36\%), MHO (31.93\%), and MAO (39.67\%). LNI, extracapsular extension, and the surgical margin were not associated with the BMI or metabolic status in each of the four groups.

A univariable analysis was performed to investigate the risk factors for the pathological features of $\mathrm{PCa}$ (Supplementary Table 1). The PSA level, biopsy GS, and clinical stage were risk factors for pathological features. Multivariate analyses were performed to ascertain whether different obesity and metabolic statuses were independently associated with the risk of pathological features of $\mathrm{PCa}$ (Table 2). The prostatectomy GS was classified into two groups of $\geq 8$ and $\leq 7$. The pathological stage was categorized into locally advanced PCa (pT3-4) and localized PCa (pT2). After adjusting for potential confounders including age, smoking status, PSA level, biopsy GS, and clinical stage, we found that MAO was associated with a significantly greater risk of a high prostatectomy GS (OR, 1.907; 95\% CI, 1.144-3.182; $P=0.013)$ and pathological stage (OR, $1.606 ; 95 \% \mathrm{CI}, 1.035-2.493 ; P=0.035)$. In contrast, the OR was not elevated in the MHO or MANW group. Inconsistent with the outcome of the pathological stage, the risk of seminal vesicle invasion in the MAO group was 1.673 times higher than that in the MHNW group (OR, 1.673; 95\% CI, 1.041-2.687; $P=0.033$ ). Neither an abnormal weight nor metabolic abnormality increased the risk of seminal vesicle invasion in the MANW group (OR, 1.094; 95\% CI, 0.643-1.860 ) or MHO group (OR, 0.726; 95\% CI, 0.478-1.104), in contrast to the MHNW group (Figure 2). Metabolic abnormality increased the possibility of LNI (OR, 1.969; 95\% CI, 1.002-3.871) in patients with normal weight, but not in patients with obesity. There were no obvious relationships of the BMI and metabolic status with extracapsular extension and surgical margins among the four groups (Table 2).

\section{Discussion}

To our knowledge, this is the first study to examine the combined effects of obesity and metabolic dysfunction on the pathological features of PCa in Eastern China. This study showed that patients with both a metabolic abnormality and obesity had a higher prostatectomy GS $(\geq 8)$ and pathological stage (pT3-4). In contrast, neither an abnormal weight nor metabolic abnormality alone increased the pathological outcomes of PCa, in contrast to MHNW.

The association between $\mathrm{PCa}$ and obesity remains controversial. One meta-analysis showed that obesity was an additional risk factor for aggressive $\mathrm{PCa}^{7}$ However, a generalized obesity index such as the BMI cannot fully reflect the risk of obesity-related metabolic complications. ${ }^{21}$ MetS is characterized by a cluster of abnormal biochemical factors. ${ }^{3}$ Several studies have investigated the relationship between MetS and worsening of the pathological features of $\mathrm{PCa}$, such as a higher GS and pT, positive surgical margin, and $\mathrm{LNI}^{3,11,12}$; however, no significant association of MetS and the GS with high-grade PCa has been found in other studies. $^{22,23}$ There is no consensus on the relationship of MetS and its individual components with $\mathrm{PCa}$ progression. Recent interest has been focused on individuals with specific BMI-metabolic status phenotypes such as MHO, MANW, and MAO, which appear to deviate from the linear 
Table I Baseline Characteristics of the 1016 Prostate Cancer Patients Undergoing Radical Prostatectomy

\begin{tabular}{|c|c|c|c|c|c|}
\hline & MHNW (\%) & MANW (\%) & MHO (\%) & MAO (\%) & $P$ value \\
\hline & 551 & 106 & 238 & $12 \mid$ & \\
\hline Age (years) & & & & & 0.110 \\
\hline$<68$ & $273(49.5)$ & $5 I(48 . I)$ & $126(52.9)$ & $74(61.2)$ & \\
\hline$\geq 68$ & $278(50.5)$ & 55 (5I.9) & $112(47.1)$ & 47 (38.8) & \\
\hline Smoking status clinical & & & & & 0.419 \\
\hline Never & $370(67.2)$ & $71(67.0)$ & $150(63.0)$ & $73(60.3)$ & \\
\hline Ever/Current & $|8|(32.8)$ & $35(33.0)$ & $88(37.0)$ & $48(39.7)$ & \\
\hline PSA (ng/mL) & & & & & 0.021 \\
\hline$<10$ & $189(34.3)$ & $32(30.2)$ & $57(23.9)$ & $27(22.3)$ & \\
\hline $10 \leq \mathrm{PSA}<20$ & $175(3 \mid .8)$ & $32(30.2)$ & $87(36.6)$ & $38(31.4)$ & \\
\hline$\geq 20$ & 187 (33.9) & $42(39.6)$ & $94(39.5)$ & $56(46.3)$ & \\
\hline Biopsy GS & & & & & 0.520 \\
\hline$\leq 6$ & $176(31.9)$ & $31(29.2)$ & $86(36.1)$ & $37(30.6)$ & \\
\hline 7 & $201(36.5)$ & $33(31.1)$ & $77(32.4)$ & $40(33.1)$ & \\
\hline$\geq 8$ & $174(3 \mid .6)$ & $42(39.6)$ & $75(31.5)$ & $44(36.4)$ & \\
\hline Clinical stage & & & & & 0.278 \\
\hline$\leq \mathrm{cT} 2 \mathrm{a}$ & $273(49.5)$ & $44(4 I .5)$ & $105(44.1)$ & $62(5 \mid .2)$ & \\
\hline $\mathrm{cT} 2 \mathrm{~b}$ & $149(27.0)$ & $31(29.2)$ & $59(24.8)$ & $31(25.6)$ & \\
\hline$\geq \mathrm{cT} 2 \mathrm{c}$ & $129(23.4)$ & $31(29.2)$ & $74(31.1)$ & $28(23.1)$ & \\
\hline Prostatectomy GS & & & & & 0.063 \\
\hline$\leq 6$ & $99(18.0)$ & $18(17.0)$ & $49(20.6)$ & $18(14.9)$ & \\
\hline 7 & $307(55.7)$ & $59(55.7)$ & II $3(47.5)$ & $55(45.5)$ & \\
\hline$\geq 8$ & $145(26.3)$ & $29(27.4)$ & $76(31.9)$ & $48(39.7)$ & \\
\hline Pathological stage & & & & & 0.005 \\
\hline pT2 & $357(64.8)$ & $65(61.3)$ & $162(68.1)$ & $60(49.6)$ & \\
\hline рT3-4 & $194(35.2)$ & $4 \mid(38.7)$ & $76(31.9)$ & $61(50.4)$ & \\
\hline Seminal vesicle invasion & & & & & 0.005 \\
\hline No & 435 (78.9) & $79(74.5)$ & $195(81.9)$ & $80(66.1)$ & \\
\hline Yes & $116(21.1)$ & $27(25.5)$ & $43(18.1)$ & $41(33.9)$ & \\
\hline Lymph node involvement & & & & & 0.092 \\
\hline No & $5 I I(92.7)$ & 91 (85.8) & $213(89.5)$ & $108(89.3)$ & \\
\hline Yes & $40(7.3)$ & $15(14.2)$ & $25(10.5)$ & $13(10.7)$ & \\
\hline Extracapsular extension & & & & & 0.095 \\
\hline No & $4 \mid 4(75.1)$ & $72(67.9)$ & $186(78.2)$ & $83(68.6)$ & \\
\hline Yes & $137(24.9)$ & $34(32.1)$ & $52(21.8)$ & $38(31.4)$ & \\
\hline Surgical margin & & & & & 0.996 \\
\hline No & $467(84.8)$ & $89(84.0)$ & $202(84.9)$ & $102(84.3)$ & \\
\hline Yes & $84(15.2)$ & $17(16.0)$ & $36(15.1)$ & $19(15.7)$ & \\
\hline Raised FPG & & & & & 0.000 \\
\hline No & $528(95.8)$ & $54(50.9)$ & $222(93.3)$ & $53(43.8)$ & \\
\hline Yes & $23(4.2)$ & $52(49.1)$ & $16(6.7)$ & $68(56.2)$ & \\
\hline Raised blood pressure & & & & & 0.000 \\
\hline No & $446(80.9)$ & $24(22.6)$ & 176 (73.9) & 51 (42.I) & \\
\hline Yes & $105(19.1)$ & $82(77.4)$ & $62(26.1)$ & $70(57.9)$ & \\
\hline
\end{tabular}

(Continued) 
Table I (Continued).

\begin{tabular}{|c|c|c|c|c|c|}
\hline & MHNW (\%) & MANW (\%) & MHO (\%) & MAO (\%) & $P$ value \\
\hline & 551 & 106 & 238 & 121 & \\
\hline $\begin{array}{l}\text { Raised TG in } \mathrm{mg} / \mathrm{dL} \\
\text { No } \\
\text { Yes }\end{array}$ & $\begin{array}{l}477(86.6) \\
74(13.4)\end{array}$ & $\begin{array}{l}43(40.6) \\
63(59.4)\end{array}$ & $\begin{array}{l}20 \mathrm{I}(84.5) \\
37(15.5)\end{array}$ & $\begin{array}{l}42(34.7) \\
79(65.3)\end{array}$ & 0.000 \\
\hline $\begin{array}{l}\text { Reduced HDL-cholesterol } \\
\text { No } \\
\text { Yes } \\
\text { Body mass index }{ }^{\mathrm{a}}\end{array}$ & $\begin{array}{l}527(95.6) \\
24(4.4) \\
22.4(21.3-23.9)\end{array}$ & $\begin{array}{l}65(61.3) \\
41(38.7) \\
23.0(21.8-24.3)\end{array}$ & $\begin{array}{l}227(95.4) \\
\text { II }(4.6) \\
26.5(25.4-28.0)\end{array}$ & $\begin{array}{l}78(64.5) \\
43(35.5) \\
26.5(25.5-28.1)\end{array}$ & 0.000 \\
\hline
\end{tabular}

Note: ${ }^{a}$ Data are median (interquartile range).

Abbreviations: PSA, prostate-specific antigen; GS, Gleason score; FPG, fasting plasma glucose; TG, triglyceride; HDL, high-density lipoprotein.

relationship between the BMI or metabolic status and its adverse clinical outcomes. ${ }^{21}$

The MHO and MANW statuses have different effects on cancer and CVD. MANW has been considered a high-risk group for CVD, all-cause mortality, dementia, and cancer, but individuals with $\mathrm{MHO}$ are not at higher risk of these conditions than are individuals with MHNW. ${ }^{16,17,21,24-28}$ Hamer et al found that metabolic risk factors were more important predictors of CVD than obesity because of the high prevalence of systemic inflammation. ${ }^{26}$ Several studies have shown that the presence of metabolic abnormalities, regardless of obesity, is an independent risk factor for colorectal neoplasm in men, suggesting that poor metabolic health plays a crucial role in the development of disease. ${ }^{16,17,27}$ However, individuals with MHO do not show a higher risk of CVD and colorectal neoplasm than do individuals with MHNW. ${ }^{16,17,26,27}$ A large prospective cohort study based on adults in the Framingham study showed that metabolic health reduced the risk of obesityrelated cancers compared with metabolic dysfunction among overweight and obese individuals. ${ }^{29}$ The preserved insulin sensitivity, relatively low visceral fat mass, and normal adipose tissue function in individuals with $\mathrm{MHO}$ might explain these different outcomes. ${ }^{30}$ In the present study, the MANW and MHO phenotypes were not associated with a greater risk of higher pathological stage and seminal vesicle invasion than was the MHNW phenotype. The incidence of aggressive PCa showed an increasing trend (Figure 2). A possible reason for the inconsistencies of MANW might be that the short follow-up did not reveal the future risk; ${ }^{26}$ alternatively, obesity and metabolic disorders acted synergistically to increase the pathological risk

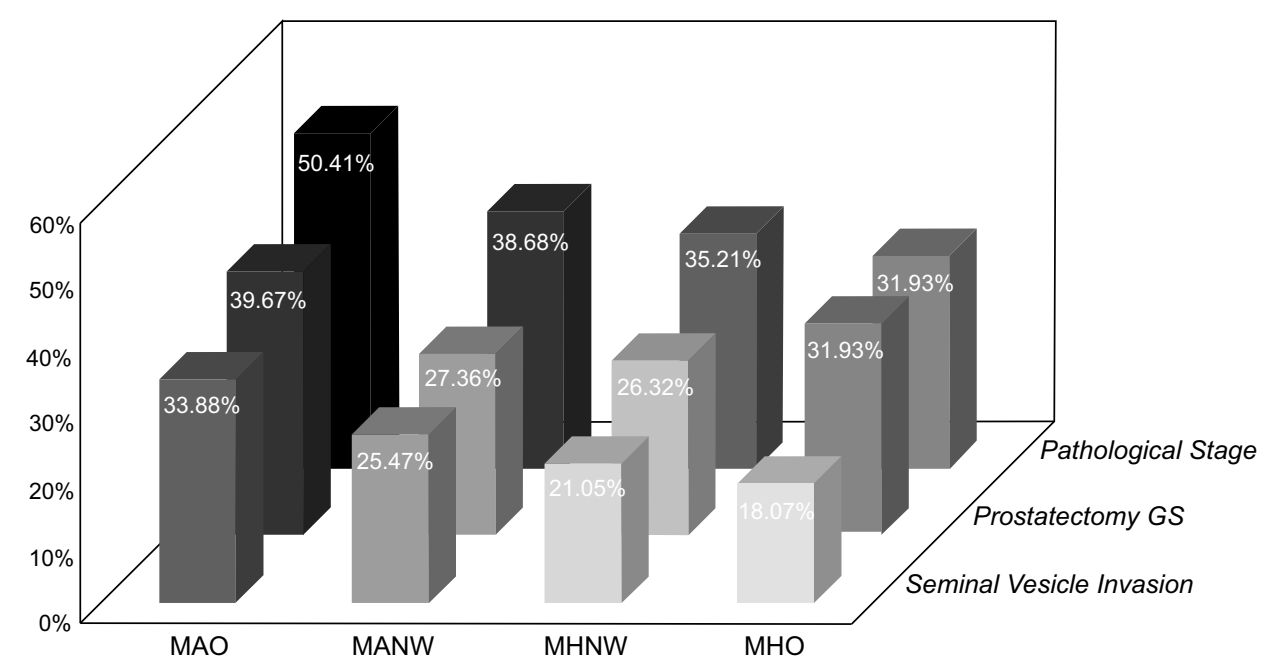

Figure I Percentages of prostatectomy GS, pathological stage, and seminal vesicle invasion in four metabolic obesity phenotypes defined by the presence of metabolic syndrome and the body mass index.Abbreviation: GS, Gleason score. 
Table 2 Metabolic Obesity Phenotypes Defined by Presence of MetS and BMI in Relation to Pathological Risk of Prostate Cancer

\begin{tabular}{|c|c|c|c|c|c|}
\hline & $\begin{array}{l}\text { MHNW } \\
\text { OR }\end{array}$ & $\begin{array}{l}\text { MHO } \\
\text { OR }(95 \% \mathrm{Cl})\end{array}$ & $\begin{array}{l}\text { MANW } \\
\text { OR }(95 \% \mathrm{Cl})\end{array}$ & $\begin{array}{l}\text { MAO } \\
\text { OR }(95 \% \mathrm{Cl})\end{array}$ & $P$ value \\
\hline \multicolumn{6}{|c|}{$\begin{array}{l}\text { Prostatectomy GS } \\
(\geq 8 \text { vs } \leq 7)\end{array}$} \\
\hline Crude & I (ref) & $1.314(0.942-1.83 \mid)$ & $1.055(0.661-1.683)$ & $1.84 \mid(1.22 I-2.776)$ & 0.004 \\
\hline Adjusted $^{\mathrm{a}}$ & I (ref) & I.467 (0.978-2.20I) & $0.802(0.462-1.390)$ & $1.907(1.144-3.182)$ & 0.013 \\
\hline \multicolumn{6}{|c|}{$\begin{array}{l}\text { Pathological stage } \\
\text { (pT3-4 vs pT2) }\end{array}$} \\
\hline Crude & I (ref) & $0.863(0.625-1.193)$ & I.I6I (0.756-I.78I) & I.87I (I.258-2.782) & 0.002 \\
\hline Adjusted $^{\mathrm{a}}$ & I (ref) & $0.767(0.538-1.093)$ & $1.022(0.639-1.634)$ & $1.606(1.035-2.493)$ & 0.035 \\
\hline \multicolumn{6}{|c|}{$\begin{array}{l}\text { Seminal vesicle invasion } \\
\text { (Yes vs No) }\end{array}$} \\
\hline Crude & I (ref) & $0.827(0.56 \mathrm{I}-\mathrm{I} .220)$ & $1.282(0.79 \mid-2.077)$ & $1.922(1.252-2.905)$ & 0.003 \\
\hline Adjusted $^{\mathrm{a}}$ & I (ref) & $0.726(0.478-1.104)$ & $1.094(0.643-1.860)$ & $1.673(1.04 I-2.687)$ & 0.033 \\
\hline \multicolumn{6}{|c|}{$\begin{array}{l}\text { LN involvement } \\
\text { (Yes vs No) }\end{array}$} \\
\hline Crude & I (ref) & $1.499(0.887-2.534)$ & $2.106(1.117-3.969)$ & $1.538(0.795-2.973)$ & 0.201 \\
\hline Adjusted $^{\mathrm{a}}$ & I (ref) & I.50I $(0.862-2.6 \mid 2)$ & $1.969(1.002-3.87 I)$ & $1.257(0.627-2.517)$ & 0.519 \\
\hline \multicolumn{6}{|c|}{$\begin{array}{l}\text { Extracapsular extension } \\
\text { (Yes vs No) }\end{array}$} \\
\hline Crude & I (ref) & $0.845(0.588-1.215)$ & I.427 (0.909-2.24I) & $1.384(0.900-2.126)$ & 0.139 \\
\hline Adjusted $^{\mathrm{a}}$ & I (ref) & $0.778(0.532-1.139)$ & $1.327(0.825-2.135)$ & $1.150(0.730-1.81 \mathrm{I})$ & 0.546 \\
\hline \multicolumn{6}{|c|}{$\begin{array}{l}\text { Surgical margin } \\
\text { (Yes vs No) }\end{array}$} \\
\hline Crude & I (ref) & $0.99 \mid(0.648-|.5| 4)$ & $1.062(0.602-1.875)$ & $1.036(0.602-1.78 I)$ & 0.899 \\
\hline Adjusted $^{\mathrm{a}}$ & I (ref) & $0.895(0.576-1.392)$ & $0.924(0.510-1.672)$ & $0.852(0.484-1.499)$ & 0.578 \\
\hline
\end{tabular}

Note: ${ }^{a}$ Adjusted for age, smoking status, PSA level, biopsy GS and clinical stage.

Abbreviations: MetS, metabolic syndrome; BMI, body mass index; PSA, prostate-specific antigen; GS, Gleason score; LN, lymph node; OR, odd ratio; Cl, confidence interval.

of PCa. Nevertheless, the metabolic abnormality and obesity phenotype is associated with aggressive $\mathrm{PCa}$, and controlling the BMI and metabolic status is therefore necessary to prevent aggressive $\mathrm{PCa}$ in individuals with $\mathrm{MHNW}$, MANW, or MHO.

Many studies have investigated the influence of specific metabolic obesity phenotypes on cancers, such as colorectal cancer and breast cancer. ${ }^{15,16,29,31}$ Kim et al were the first to explore the incidence of PCa according to the BMI and metabolic status. ${ }^{32}$ Both obesity and metabolic disorders increased the risk of $\mathrm{PCa}$, and the risk increased according to the number of components of MetS. However, they found that the presence of a metabolic disease was more important than the BMI. We further analyzed the effect of obesity and the metabolic status on the adverse pathological features of PCa. The PSA level, biopsy GS, and clinical stage were associated with the progression of PCa (Supplementary Table 1); therefore, we adjusted for these factors in the multivariable analyses. Based on MHNW, only individuals with both obesity and MetS had a significantly elevated risk of a high prostatectomy GS (OR, 1.907) and pathological stage (OR, 1.606), which were associated with disease progression. In contrast, neither an abnormal weight nor metabolic abnormality alone was associated with a higher GS or stage. The effect of either obesity or a metabolic abnormality on pathology was not significant, suggesting that obesity or MetS by itself may not be an independent risk factor for aggressive PCa. Obesity and metabolic disorders might act synergistically to increase the pathological risk of PCa. Interestingly, the risk of seminal vesicle invasion in the MAO group was 1.67 times higher than that in the MHNW group, which is inconsistent with the outcome of the pathological stage (Figure 2). Seminal vesicle invasion might be more easily affected by obesity and metabolic abnormalities than LNI and extracapsular extension. Patients with a normal weight and metabolic abnormality had a higher risk of LNI 

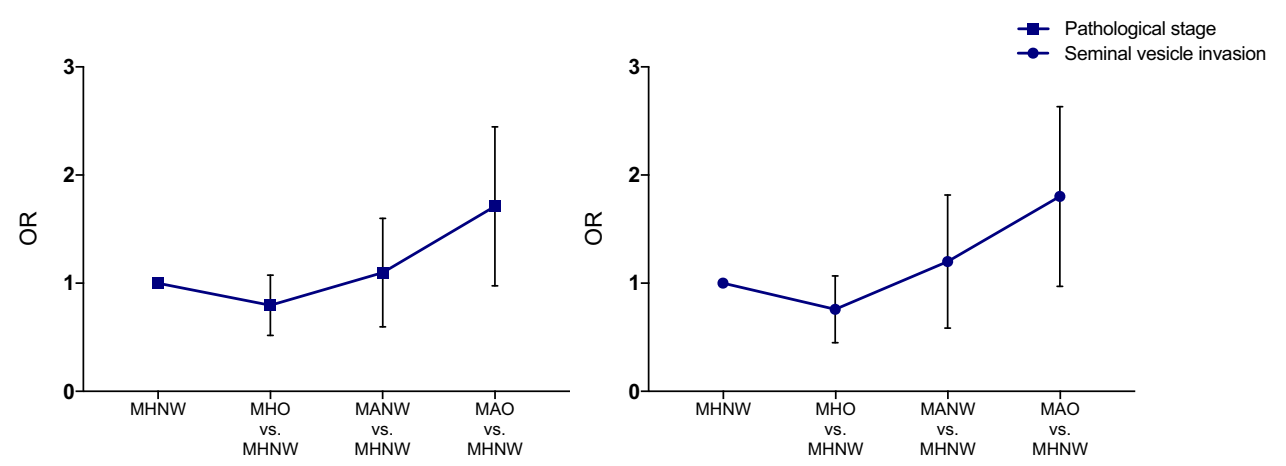

Figure 2 ORs of pathological stage and seminal vesicle invasion of prostate cancer calculated with the MHNW group as a reference group.Abbreviations: OR, odds ratio; MHNW, metabolically healthy and normal weight.

(OR, 1.969), but patients with obesity did not. Controlling metabolic disease might help to decrease the incidence of LNI of PCa after prostatectomy. The BMI and metabolic status do not influence the risk of extracapsular extension or positive surgical margins.

Theoretical biologic mechanisms for the positive relationship of the pathological risk of PCa with obesity and metabolic disorders are complex. The key mechanism linking obesity and MetS to an increased risk of cancer has been proposed to be insulin resistance. ${ }^{33}$ Several biologic hypotheses for PCa aggressiveness in relation to MetS have been proposed. First, high insulin and insulin-like growth factor-1 levels in patients with insulin resistance are associated with the growth of both androgen-sensitive and androgenindependent human $\mathrm{PCa}$ cell lines in vitro. ${ }^{34}$ Second, changes in the sex steroid pathway, including increased estradiol levels and decreased free testosterone levels, promote the differentiation of PCa cells, resulting in more aggressive $\mathrm{PCa}^{34-36}$ Third, obesity disrupts the dynamic role of adipocytes in energy homeostasis, resulting in inflammatory cytokine secretion and alteration of adipokines (increased leptin and decreased adiponectin levels), which can promote epithelial cell proliferation and stimulate angiogenesis. $^{37,38}$

To the best of our knowledge, this is the first study to compare the pathological risk of $\mathrm{PCa}$ among $\mathrm{MHNW}, \mathrm{MHO}$, MANW, and MAO phenotypes in Chinese men. Nevertheless, this study has several limitations. First, although the statuses of the BMI and metabolic disorders change over time, they were evaluated only once in this study. Therefore, the influence of dynamic changes in the body weight and metabolic status on the risk of PCa could not be assessed. Further longitudinal studies are needed to identify the relationship of changes in the BMI and metabolic status with the pathological risk of PCa. Second, because of the inherent limitations of a cross-sectional study design, it was not possible to clarify a causal relationship between different metabolic obesity phenotypes and the risk of PCa. Third, our study was a retrospective, hospital-based study rather than a population-based study; this might have resulted in some degree of selection bias.

\section{Conclusion}

We demonstrated that the MAO phenotype was associated with aggressive PCa, including a higher prostatectomy GS, pathological stage, and seminal vesicle invasion. In contrast, neither the MHO nor MANW phenotype was associated with high-risk pathological features of PCa. Obesity and metabolic disorders act synergistically to increase the pathological risk. These findings indicate that controlling the BMI and metabolic status is necessary to prevent aggressive $\mathrm{PCa}$ and progression of $\mathrm{PCa}$.

\section{Acknowledgments}

We thank Angela Morben, DVM, ELS, from Liwen Bianji, Edanz Editing China, for editing the English text of a draft of this manuscript. This study was partly funded by the National Natural Science Foundation of China (Grant No. NSFC 81502195).

\section{Disclosure}

The authors report no conflicts of interest in this work.

\section{References}

1. Bray F, Ferlay J, Soerjomataram I, et al. Global cancer statistics 2018: GLOBOCAN estimates of incidence and mortality worldwide for 36 cancers in 185 countries. CA Cancer J Clin. 2018;68(6):394-424. doi:10.3322/caac. 21492 
2. National Health Commission of People's Republic of China. Chinese guidelines for diagnosis and treatment of prostate cancer 2018 (English version). Chin $J$ Cancer Res. 2019;31(1):67-83. doi:10.21147/j.issn.1000-9604.2019.01.04

3. Zhang GM, Zhu Y, Dong DH, et al. The association between metabolic syndrome and advanced prostate cancer in Chinese patients receiving radical prostatectomy. Asian $J$ Androl. 2015;17 (5):839-844. doi:10.4103/1008-682X.148138

4. Leitzmann MF, Rohrmann S. Risk factors for the onset of prostatic cancer: age, location, and behavioral correlates. Clin Epidemiol. 2012;4:1-11. doi:10.2147/CLEP.S16747

5. Lund Haheim L, Wisloff TF, Holme I, Nafstad P. Metabolic syndrome predicts prostate cancer in a cohort of middle-aged Norwegian men followed for 27 years. Am J Epidemiol. 2006;164(8):769-774. doi:10.1093/aje/kwj284

6. Arthur R, Moller H, Garmo H, et al. Serum glucose, triglycerides, and cholesterol in relation to prostate cancer death in the Swedish AMORIS study. Cancer Causes Control. 2019;30(2):195-206. doi:10.1007/s10552-018-1093-1

7. Zhang X, Zhou G, Sun B, et al. Impact of obesity upon prostate cancer-associated mortality: a meta-analysis of 17 cohort studies. Oncol Lett. 2015;9(3):1307-1312. doi:10.3892/ol.2014.2841

8. Liang Z, Xie B, Li J, et al. Hypertension and risk of prostate cancer: a systematic review and meta-analysis. Sci Rep. 2016;6:31358. doi:10.1038/srep31358

9. Jian Gang P, Mo L, Lu Y, Runqi L, Xing Z. Diabetes mellitus and the risk of prostate cancer: an update and cumulative meta-analysis. Endocr Res. 2015;40(1):54-61. doi:10.3109/07435800.2014.934961

10. YuPeng L, YuXue Z, PengFei L, et al. Cholesterol levels in blood and the risk of prostate cancer: a meta-analysis of 14 prospective studies. Cancer Epidemiol Biomarkers Prev. 2015;24(7):1086-1093. doi:10.1158/1055-9965.EPI-14-1329

11. Morlacco A, Dal Moro F, Rangel LJ, et al. Impact of metabolic syndrome on oncologic outcomes at radical prostatectomy. Urol Oncol. 2018;36(12):528.e521-528.e526. doi:10.1016/j. urolonc.2018.10.003

12. Bhindi B, Xie WY, Kulkarni GS, et al. Influence of metabolic syndrome on prostate cancer stage, grade, and overall recurrence risk in men undergoing radical prostatectomy. Urology. 2016;93:77-85. doi:10.1016/j.urology.2016.01.041

13. Zhang GM, Qin XJ, Zhang HL, et al. Serum lipid profiles: novel biomarkers predicting advanced prostate cancer in patients receiving radical prostatectomy. Asian $J$ Androl. 2015;17(2):239-244. doi:10.4103/1008-682X.142135

14. Jung $\mathrm{CH}$, Lee WJ, Song KH. Metabolically healthy obesity: a friend or foe? Korean J Intern Med. 2017;32(4):611-621. doi:10.3904/ kjim.2016.259

15. Park YM, White AJ, Nichols HB, et al. The association between metabolic health, obesity phenotype and the risk of breast cancer. Int J Cancer. 2017;140(12):2657-2666. doi:10.1002/ijc.30684

16. Kim NH, Jung YS, Park JH, Park DI, Sohn CI. Influence of obesity and metabolic abnormalities on the risk of developing colorectal neoplasia. Dig Dis Sci. 2018;63(11):3126-3133. doi:10.1007/ s10620-018-5239-5

17. Kim M-C, Kim C-S, Chung T-H, et al. MONW phenotype is associated with advanced colorectal adenoma in Korean men. Obesity (Silver Spring). 2012;20(9):1876-1881. doi:10.1038/oby.2011.34

18. Kim JY, Park DI, Yu J, et al. Increased risk of advanced colorectal neoplasia among Korean men with metabolic abnormality and obesity. Clin Gastroenterol Hepatol. 2016;14(9):1310-1316.e1312. doi:10.1016/j.cgh.2016.04.022

19. Chinese Diabetes Society. Guidelines for the prevention and control of type 2 diabetes in China (2017 Edition). Chin J Diabetes Mellit. 2018;10(1):4-67.
20. Who. Obesity: preventing and managing the global epidemic: report of a WHO. Report of the World Health Organization Consultation of Obesity. 2000;894.

21. Yoo HJ, Hwang SY, Hong HC, et al. Association of metabolically abnormal but normal weight (MANW) and metabolically healthy but obese (MHO) individuals with arterial stiffness and carotid atherosclerosis. Atherosclerosis. 2014;234(1):218-223. doi:10.1016/ j.atherosclerosis.2014.02.033

22. Beebe-dimmer JL, Nock NL, Neslund-dudas C, et al. Racial differences in risk of prostate cancer associated with metabolic syndrome. Urology. 2009;74(1):185-190. doi:10.1016/j.urology.2009.03.013

23. Jeon KP, Jeong TY, Lee SY, et al. Prostate cancer in patients with metabolic syndrome is associated with low grade Gleason score when diagnosed on biopsy. Korean $J$ Urol. 2012;53(9):593-597. doi:10.4111/kju.2012.53.9.593

24. Stefan N, Kantartzis K, Machann J, et al. Identification and characterization of metabolically benign obesity in humans. Arch Intern Med. 2008;168(15):1609-1616. doi:10.1001/archinte.168.15.1609

25. Ortega FB, Lee DC, Katzmarzyk PT, et al. The intriguing metabolically healthy but obese phenotype: cardiovascular prognosis and role of fitness. Eur Heart J. 2013;34(5):389-397. doi:10.1093/eurheartj/ ehs 174

26. Hamer M, Stamatakis E. Metabolically healthy obesity and risk of all-cause and cardiovascular disease mortality. J Clin Endocrinol Metab. 2012;97(7):2482-2488. doi:10.1210/jc.2011-3475

27. Ko SH, Baeg MK, Ko SY, et al. Obesity and metabolic unhealthiness have different effects on colorectal neoplasms. J Clin Endocrinol Metab. 2017;102(8):2762-2769. doi:10.1210/jc.2017-00152

28. Lee JY, Han K, Han E, et al. Risk of incident dementia according to metabolic health and obesity status in late life: a population-based cohort study. J Clin Endocrinol Metab. 2019;104(7):2942-2952. doi:10.1210/jc.2018-01491

29. Moore LL, Chadid S, Singer MR, Kreger BE, Denis GV. Metabolic health reduces risk of obesity-related cancer in framingham study adults. Cancer Epidemiol Biomarkers Prev. 2014;23(10):2057-2065. doi:10.1158/1055-9965.EPI-14-0240

30. Bluher M. The distinction of metabolically 'healthy' from 'unhealthy' obese individuals. Curr Opin Lipidol. 2010;21 (1):38-43. doi:10.1097/MOL.0b013e3283346ccc

31. Akinyemiju T, Moore JX, Pisu M, et al. A prospective study of obesity, metabolic health, and cancer mortality. Obesity (Silver Spring). 2018;26(1):193-201. doi:10.1002/oby.22067

32. Kim JW, Ahn ST, Oh MM, et al. Incidence of prostate cancer according to metabolic health status: a nationwide cohort study. J Korean Med Sci. 2019;34(6):e49. doi:10.3346/jkms.2019.34.e49

33. Kopp W. High-insulinogenic nutrition-an etiologic factor for obesity and the metabolic syndrome? Metabolism. 2003;52(7):840-844. doi:10.1016/S0026-0495(02)05294-0

34. De Nunzio C, Aronson W, Freedland SJ, Giovannucci E, Parsons JK. The correlation between metabolic syndrome and prostatic diseases. Eur Urol. 2012;61(3):560-570. doi:10.1016/j.eururo.2011.11.013

35. Buschemeyer WC 3rd, Freedland SJ. Obesity and prostate cancer: epidemiology and clinical implications. Eur Urol. 2007;52 (2):331-343. doi:10.1016/j.eururo.2007.04.069

36. Freedland SJ. Obesity and prostate cancer: a growing problem. Clin Cancer Res. 2005;11(19 Pt 1):6763-6766. doi:10.1158/1078-0432. CCR-05-1305

37. Khandekar MJ, Cohen P, Spiegelman BM. Molecular mechanisms of cancer development in obesity. Nat Rev Cancer. 2011;11 (12):886-895. doi:10.1038/nrc3174

38. De Marzo AM, Platz EA, Sutcliffe S, et al. Inflammation in prostate carcinogenesis. Nat Rev Cancer. 2007;7(4):256-269. doi:10.1038/ $\operatorname{nrc} 2090$ 


\section{Publish your work in this journal}

Cancer Management and Research is an international, peer-reviewed open access journal focusing on cancer research and the optimal use of preventative and integrated treatment interventions to achieve improved outcomes, enhanced survival and quality of life for the cancer patient.
The manuscript management system is completely online and includes a very quick and fair peer-review system, which is all easy to use. Visit http://www.dovepress.com/testimonials.php to read real quotes from published authors.

Submit your manuscript here: https://www.dovepress.com/cancer-management-and-research-journal 